\title{
THE ROLE OF OBSERVATION WITH HORIZONTAL MERIDIAN CIRCLE IN CHINA FOR ESTABLISHING AN INERTIAL FRAME
}

\author{
Li Zhi-gang and Qi GuAN-Rong \\ Shaanxi Astronomical Observatory \\ Lintong, Xian, China, P.R.
}

ABSTRACT. While HIPPARCOS is expected to measure positions and proper motions with more accuracy than those obtained by ground-based instruments, what can we do in the future for groundbased instruments? The observations with them still are important for establishing an inertial frame because of the long history of observations with them and improvements in the instruments. Moreover, it is necessary to have data of observations from them for research on problems related to the Earth. The horizontal meridian circle in China (DCMT) is expected to have advantage over the classical meridian circles. The DCMT will be assembled and tested this year. It should work in the following fields: (1) observing radio stars, (2) observation of minor planets, (3) absolute determinations of IRS.

\section{ON THE PROBLEM OF THE DEVELOPMENT OF ASTRONOMICAL REFRACTION METHODOLOGY ON THE BASIS OF NEW TECHNOLOGY}

\author{
V.I. SERGIENKo \\ NPO Etalon \\ Borodina 57 \\ 664018 Irkutsk, USSR
}

ABSTRACT. The work on construction and operation of automated systems for collection and processing astrometric and meteorological information and also of automated television systems with remote control have placed before us a number of fundamental difficulties in that hundreds of correct solutions to these problems require a systematic approach.

The most suitable cybernetics-mathematical composite for solving the problems of astronomical refraction may become the so-called hybrid-expert systems - a union of traditional expert systems with calculation-logical ones. In these systems logical-linguistic models are used together with mathematical ones.

The basic problem of ARA-C consists in obtaining a model of the environment in which light propagates and also to give a notion of interaction between electrons (environment) and photons (light).

It is proposed to use in this system a model of light interaction with environment built up on special formalisms of artificial intellect (logical-linguistic model). The basic principal in hybridexpert system ARA-C operation must be mathematical modelling and calculation experimentation. In the proposed hybrid-expert system knowledge is presented on three levels - object, mathematical and programmed. Functioning of the lower levels of ARA-C is connected with formulation of the calculation problems. A calculation problem presented to a technical model determines a partial order on a twopart column of mathematical correlations 